\title{
Strong Eddy-Current Shielding of Ferromagnetic Resonance Response in Sub-Skin-Depth-Thick Conducting Magnetic Multilayers
}

\author{
Ivan S. Maksymov ${ }^{1}$, Zhaoyang Zhang ${ }^{1,2}$, Crosby Chang ${ }^{1}$ and Mikhail Kostylev ${ }^{1}$ \\ ${ }^{1}$ School of Physics, M013, University of Western Australia, Crawley WA 6009, Australia \\ ${ }^{2}$ University of Science and Technology of China, Hefei, China
}

Received 1 April 2010, revised 15 April 2010, accepted 20 April 2010, published 1 June 2010 (dates will be inserted by IEEE).

Abstract-Exchange-coupled nonmagnetic metal (NM) and ferromagnetic metal (FM) multilayers are crucial for microwave magnonic and spintronic devices. These layered materials usually have total thicknesses smaller than the microwave skin depth. By using a stripline broadband ferromagnetic resonance (BFMR) spectroscopy technique, we experimentally demonstrate that the amplitude of the magnetization precession in the FM layer is strongly diminished by the shielding effect of microwave $(6-12 \mathrm{GHz})$ eddy currents circulating in the NM capping layers.

Index Terms-Microwave magnetics, eddy currents, thin films, metamaterials

\section{INTRODUCTION}

Magnonics and spintronics are emerging nanotechnologies enabling nonvolatile memory and logics, spin-torque nano-oscillators [Kruglyak 2010, Stamps 2014], left-handed metamaterials [Wu 2005, Chen 2006], and safer gas sensors [Chang 2013]. These devices operate at microwave frequencies and they often consist of nonmagnetic metal (NM) and ferromagnetic metal (FM) multilayers.

Permalloy $\left(\mathrm{Py}=\mathrm{Ni}_{80} \mathrm{Fe}_{20}\right)$ is the material of choice for the FM layer. It exhibits, e.g., an optimal combination of magnetic properties such as the vanishing magnetic anisotropy and one of the smallest magnetic (Gilbert) losses $\alpha_{G}$ among FMs [Kruglyak 2010]. NM layers are usually made of $\mathrm{Ta}, \mathrm{Cu}, \mathrm{Au}, \mathrm{Pt}$, or Pd. Ta and $\mathrm{Cu}$ thin films often act as a seed layer or as a capping layer protecting the Py film from oxidation [Kowalewski 2000, Kennewell 2010]. Pt and Pd layers are used in devices exploiting the spin pumping and Spin-Hall effects [Brataas 2012, Jungwirth 2012, Boone 2013], and in gas sensors [Chang 2013]. As the spin pumping effect in $\mathrm{Cu}$ layers is negligible [Mizukami 2001], multilayers with $\mathrm{NM}=\mathrm{Cu}$ are often used as reference samples [Kim 2011, Boone 2013].

The most popular way to expose a planar sample to microwave field is to employ the stripline broadband ferromagnetic resonance (BFMR) spectroscopy (see, e.g., [Silva 1999, Counil 2004, Bilzer 2007]). In this case, only one surface of the sample is exposed to microwave radiation. The BFMR spectroscopy is largely used to study the magnetization dynamics and spin current injection through interfaces (spin pumping and inverse spin Hall effects).

Corresponding author: M. Kostylev (mikhail.kostylev@uwa.edu.au). Digital Object Identifier: 10.1109/ML.2010.2010123 (inserted by IEEE).
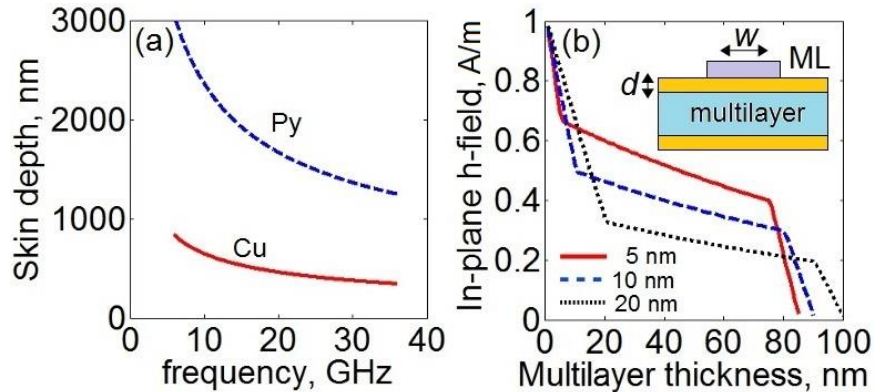

Fig. 1. (a) Theoretical skin-depth $\delta$ of $\mathrm{Cu}$ and Py as a function of the microwave frequency $f$. (b) Inset: Schematic of a NM/FM/NM multilayer with the NM capping layer of thickness $d$ facing a microstrip line $(\mathrm{ML})$ of width $w$. Calculated profiles of the in-plane component of the microwave magnetic field (m.m.f.) across the multilayers with different $\mathrm{Cu}$ capping layer thicknesses $d$. The total thickness of the multilayer is counted from the side facing the ML. $f=12 \mathrm{GHz}$ and the thickness of the Cu seed layer is $10 \mathrm{~nm}$ in all cases in Panel (b).

It is a common belief that metal layers thinner than the microwave skin-depth $\delta$ [Fig. 1(a)] do not affect the FMR response [Charilaou 2010]. However, the crucial role of the microwave eddy currents (ECs) in the formation of the FMR response of sub-skin-depth-thick FM films has been established theoretically [Chan 2000, Chen 2006, Kostylev 2009, Kennewell 2010, Maksymov 2013, Bailleul 2013, Bailey 2013, Maksymov 2014]. Numerous experiments have confirmed a strong impact of ECs [Kennewell 2010, Kostylev 2011, Kostylev 2013, Bailey 2013], and good agreement between theory and experiment was found.

In this work, we investigate NM/FM/NM multilayers and present experimental evidence of a strong and adverse effect of sub-skin-depth-thick NM $(\mathrm{Cu})$ capping layers on the strength and profile of the FMR response of the underlying FM 
(Py) layer. In agreement with theory [Fig. 1(b)], we show that ECs circulating in the capping layer shield the Py layer from the microwave magnetic field (m.m.f.) induced by the microstip line (ML) transducer of the BFMR setup.

Our findings are also applicable in situations where the m.m.f. is incident on one film surface only. The possibility of generalization was demonstrated in our recent experiment [Kostylev 2013] and confirmed theoretically [Kostylev 2012]. In stripline BFMR, samples are exposed to a near m.m.f. of a stripline. However, in [Kostylev 2012, 2013] we showed that exposure of FM films to a far m.m.f. results in the same behavior. This similarity is important, e.g., for characterization of magnetic metamaterials [Wu 2005]. Note that the far-field and near-field responses are similar, provided the stripline width is large enough, as in our experiment. The requirement of a large stripline width ensures the absence of the adverse effect of travelling spin wave contribution to the FMR response [Counil 2004] and should be fulfilled in any BFMR experiment.

\section{EXPERIMENTAL RESULTS AND DISCUSSION}

We use a $0.33 \mathrm{~mm}$-wide $\mathrm{ML}$ transducer and place the sample grown on a Si substrate on top of it. For such a wide $\mathrm{ML}$ transducer the EC shielding should be strong [Maksymov 2014]. We deliberately use a ML and not a coplanar waveguide (CPW) because M. Bailleul [2013] showed that for a CPW the microwave electric field (m.e.f.) shielding may coexist with the m.m.f. shielding (see Appendix). We carry out BFMR measurements on magnetron sputter-deposited $\mathrm{Si} / \mathrm{Cu}[10 \mathrm{~nm}] / \mathrm{Py}[70 \mathrm{~nm}] / \mathrm{Cu}[d]$ multilayers with $d=10,20,35$ and $70 \mathrm{~nm}$. The capping layer of the samples faces ML (Fig. 1). We keep the microwave frequency $f$ constant and sweep the magnetic field $H$ applied in the sample plane and along the transducer. To record the raw FMR absorption traces we use a microwave network analyzer. The measurements are taken at room temperature. Although in many experimental situations the thickness of the NM capping layers is $<10 \mathrm{~nm}, 10 \mathrm{~nm}$ thick capping layers are also often used, e.g., in Pt-YIG (yttrium-iron-garnet) magnetic structures [Sandweg 2011, Hahn 2013]. Furthermore, the impact of ECs strongly depends on the frequency (see Fig. 5 in [Kennewell 2010]). Therefore, our results for the $10 \mathrm{~nm}-$ range capping layers in the $6-12 \mathrm{GHz}$ range may be important for experiments at $\sim 40 \mathrm{GHz}$ employing samples with $d=5 \mathrm{~nm}$.

To demonstrate the impact of the EC shielding on results of BFMR measurements, we study the samples with thicknesses equal to multiples of the technologically meaningful value $d=10 \mathrm{~nm}$. Measuring samples with thicker (but still sub-skin-depth) capping layers and comparing them to the reference $d=10 \mathrm{~nm}$ sample allows us to easily establish the functional dependence of the shielding effect on the layer thickness. For the same reason we use a thick Py layer, although the theory predicts a significant impact of ECs for much thinner samples, too (see Fig. 6 in [Kostylev 2009]).

The FMR absorption spectrum of the ML is obtained as a ratio of the complex scattering parameter S21 of the loaded
$\mathrm{ML}$ (with a sample) to that of the unloaded ML (S210) [Fig. 2(a)]. One sees dips in the spectra taken at different frequencies $f$. The amplitude of these dips corresponds to the amplitude of the respective FMR response of the multilayer.
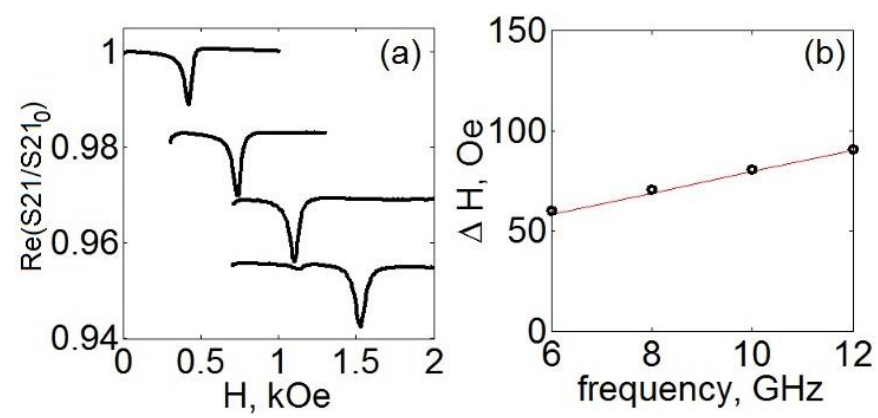

Fig. 2. (a) Experimental FMR absorption spectra of the multilayer with $d=20 \mathrm{~nm}$ for $f=6,8,10$ and $12 \mathrm{GHz}$ (from top to bottom). For clarity, each spectrum is vertically offset by -0.015 . (b) Experimental linewidth $\Delta H$ of the FMR response of the multilayer with $d=20 \mathrm{~nm}$ as a function of $f$. The straight line is the best fit of the experimental data. An extra absorption peak of small amplitude in the lowest trace of Panel (a) (at $\sim 1.1 \mathrm{kOe}$ ) is the response of the first higher-order standing spin wave mode ( $1^{\text {st }}$ SSWM) [Kostylev 2009].

Figure 3 shows the experimental and theoretical amplitudes of the FMR response. The amplitude drops very quickly as $d$ is increased. The decrease in the amplitude is due to ECs circulating in the capping layers, which shield the Py film from the m.m.f. [Maksymov 2013].

We obtain the values of the gyromagnetic ratio $\gamma /(2 \pi)$ and of the saturation magnetization for the FM layer $\left(4 \pi M_{\mathrm{s}}\right)$ by best-fitting experimental data with the Kittel formula

$$
f=\frac{|\gamma|}{2 \pi} \sqrt{H\left(H+4 \pi M_{\mathrm{s}}\right)} .
$$

These values are used to extract the values of the Gilbert damping parameter $\alpha_{\mathrm{G}}$. From the measured FMR absorption spectra we extract the full width at half maximum line width $\Delta H$ as a function of $f$ [Fig. 2(b)] and fit the obtained dependence with the formula

$$
\Delta H=\frac{2 \alpha f}{|\gamma| /(2 \pi)}+\Delta H_{0} .
$$

Table 1. Parameters extracted from experimental FMR absorption traces for different values of $d$.

\begin{tabular}{ccccc}
\hline \hline$d, \mathrm{~nm}$ & 10 & 20 & 35 & 70 \\
\hline$\gamma /(2 \pi), \mathrm{MHz} / \mathrm{Oe}$ & 2.998 & 2.996 & 3.025 & 3.030 \\
$4 \pi M_{s}, \mathrm{G}$ & 8003 & 9033 & 8676 & 8790 \\
$\alpha_{\mathrm{G}}$ & 0.0075 & 0.008 & 0.0086 & 0.0106 \\
$\Delta H(f=10 \mathrm{GHz}), \mathrm{Oe}$ & 90.7 & 80.62 & 100.8 & 120.9 \\
$\Delta H_{0}, \mathrm{Oe}$ & 40.84 & 26.44 & 33.71 & 48.95 \\
\hline \hline
\end{tabular}

The parameters extracted from experimental FMR traces are presented in Table 1. One sees that we have obtained typical values of $\gamma /(2 \pi)$ [Shaw 2013], $4 \pi M_{\mathrm{s}}$ [Kennewell 2010] 
and $\alpha_{\mathrm{G}}$ [Kruglyak 2010] for Py. For $d=10 \mathrm{~nm}$ we find $\alpha_{\mathrm{G}}=0.0075$, which is very close to the typical value for Py: 0.008 [Kruglyak 2010]. The damping parameters for the $d=10 \mathrm{~nm}$ sample are somewhat different from the other three samples. Indeed, for the remaining samples one observes a clear trend: an increase in $d$ correlates with an increase in $\alpha_{\mathrm{G}}$ and $\Delta H_{0}$. Furthermore, this trend suggests that EC may also contribute to the frequency independent part of the FMR losses $\Delta H_{0}$. The correlation between $\alpha_{\mathrm{G}}$ and the thickness of the FM film is well-known [Charilaou 2010, Maksymov 2013a]. However, in our experiment the thickness of the FM is constant but the thickness of the NM capping layer is varied. The clear correlation of the loss parameters with $d$ also suggests that the magnetic quality of the Py layer of the $d=10 \mathrm{~nm}$-thick film is somewhat different from the other films. This is seen not only from the large $\Delta H(f=10 \mathrm{GHz})$ value for it, but also from the noticeably smaller $4 \pi M_{\mathrm{s}}$ than for the other films. This may be the reason why the magnetic losses for this film do not follow this trend.

We employ our theory [Kostylev 2009] to calculate the FMR amplitude as a function of $d$. The data from Table 1 are used as the input parameters. We use the bulk $\mathrm{Cu}$ conductivity for both NM layers $\left(\sigma_{\text {bulk }}=5.96 \times 10^{7} \mathrm{~S} / \mathrm{m}\right)$ and obtain a $d$-dependence of the FMR amplitude which is significantly steeper than the experimental one. We also see that the linewidth of the simulated FMR traces strongly increases with an increase in $d$. For instance, $\Delta H(f=10 \mathrm{GHz})$ for $d=70 \mathrm{~nm}$ extracted from the simulated trace is almost 1.5 times larger than the respective value from Table 1.

Accordingly, in the next round of simulations, we decrease the value of $\sigma$ and use $\alpha_{G}$ and $\Delta H_{0}$ values for $d=20 \mathrm{~nm}$ from Table 1 for the calculations for $d=20,35$ and $70 \mathrm{~nm}$. It is known that $\sigma$ of thin $\mathrm{Cu}$ films can be lower than $\sigma_{\text {bulk }}$ [Schmiedl 2008]. This justifies the new choice of $\sigma=0.4 \sigma_{\text {bulk }}$ for the capping layers. The new result (Fig. 3 ) is in reasonably good agreement with the experiment. Importantly, the simulated raw traces show shapes very similar to those in Fig. 2(a) - characterized by small amplitude of the $1^{\text {st }}$ SSWM with respect to the fundamental mode. Note that to keep the amplitude of this mode small we need to keep the conductivity of the seed $\mathrm{Cu}$ layer equal to $\sigma_{\text {bulk. }}$. Otherwise the calculation delivers much larger relative amplitudes of the $1^{\text {st }}$ SSWM. The much larger relative amplitude is due to strong nonuniformity of the in-plane m.m.f. inside the Py layer. The nonuniformity increases with an increase in the EC density inside this layer. The smaller the conductivity of the seed layer, the larger is the EC inside the Py layer. Hence, the practically vanishing amplitude of the $1^{\text {st }}$ SSWM in the experimental traces may be considered as an evidence of a noticeably larger conductivity of the seed layer with respect to the capping layer: a large current flowing inside the seed layer makes the microwave EC field in the Py layer more uniform. Indeed, it has been shown experimentally that the conductivity of $\mathrm{Cu}$ layers strongly depends on the layer on which they grow [Speriosu 1993]. More precisely, a $\mathrm{Cu}$ layer grown on top of a Py layer may have significantly smaller conductivity than the one grown on a bare substrate [Kowalewski 2000].

Importantly, the $2^{\text {nd }}$ round of simulations delivers values of the resonance linewidth very similar to the experimental ones from Table 1. In particular, from the simulated FMR traces we extract $\Delta H(f=10 \mathrm{GHz})=107.8 \mathrm{Oe}$ and 118.9 Oe for $d=35$ and $70 \mathrm{~nm}$, respectively. This demonstrates that the significant increase in the magnetic losses seen in Table 1 for the samples with the thicker capping layers may be entirely due to the microwave eddy currents. This result also confirms the validity of our assumption of the reduced $\sigma$ for the capping layers $\left(\sigma=0.4 \sigma_{\text {bulk }}\right)$, since otherwise the simulated linewidth broadening due to the eddy currents would be much larger.

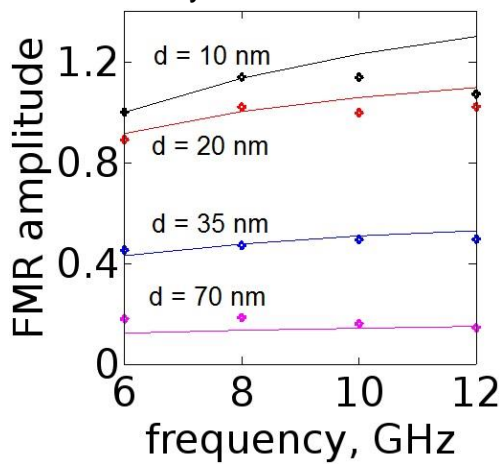

Fig. 3. Experimental (symbols) and theoretical (curves) relative amplitudes of the FMR response of the Cu-Py multilayer samples as a function of $f$. All curves are normalized to the absorption amplitude of the sample with $d=10 \mathrm{~nm}$ at $6 \mathrm{GHz}$. Some disagreement between the theory and the experiment, especially for the case of $d=10 \mathrm{~nm}$ at 10 and $12 \mathrm{GHz}$, may be attributed to the effects of imprecise sample placement on the ML and the sample dimensions [Bilzer 2007], as well as to asymmetry of the FMR lineshape [Miroshnichenko 2010], which could not be taken into account in the simulations.

\section{CONCLUSIONS}

We have experimentally investigated the broadband FMR response of metallic magnetic multilayer structures and demonstrated a crucial effect of non-magnetic metallic capping layers of sub-skin-depth thicknesses on the strength of this response. Eddy currents circulating in the capping layers shield the ferromagnetic film from the m.m.f. The shielding leads to a strong decrease in the amplitude of the FMR response and strong resonance linewidth broadening. These findings have direct implications for microwave characterization of magnetic materials, including materials for spin-transport applications. They are also important for applications of conductive ferromagnetic films in frequency agile metamaterials [Wu 2005, Chen 2006].

\section{APPENDIX}

For the ML geometry the m.e.f. shielding is not relevant because of a significant separation of the sample from the $\mathrm{ML}$ ground plane. The ML equivalent of the $s+g$ parameter for 
CPWs introduced by Bailleul [2013] is the thickness of the ML substrate. The substrate thickness is usually large which leads to a negligible contribution of the m.e.f. shielding to the total shielding effect. We confirmed the unimportance of the m.e.f. shielding for our ML transducer by rigorous finite-difference time-domain simulations. The simulations showed that the microwave current in the sample flows in the opposite direction to the one in the microstrip. This is a clear indication of the dominance of the m.m.f. shielding regime in our case.

\section{ACKNOWLEDGMENT}

This work was supported by the UWA UPRF scheme and the Australian Research Council.

\section{REFERENCES}

Bailey W E, Cheng C, Knut R, Karis O, Auffret S, Zohar S, Keavney D, Warnicke P, Lee J S, Arena D A (2013) "Detection of microwave phase variation in nanometre-scale magnetic heterostructures," Nat. Commun., vol. 4, 2025.

Bailleul M (2013), "Shielding of the electromagnetic field of a coplanar waveguide by a metal film: Implications for broadband ferromagnetic resonance measurements," Appl. Phys. Lett., vol. 103, 192405.

Bilzer C, Devolder T, Crozat P, Chappert C, Cardoso S, Freitas P P (2007), "Vector network analyzer ferromagnetic resonance of thin films on coplanar waveguides: Comparison of different evaluation methods," J. Appl. Phys., vol. 101, 074505.

Boone C T, Nembach H T, Shaw J M, Silva T J (2013), "Spin transport parameters in metallic multilayers determined by ferromagnetic resonance measurements of spin-pumping," J. Appl. Phys., vol. 113, 153906.

Brataas A, Kent A D, Ohno H (2012), "Current-induced torques in magnetic materials," Nat. Mater., vol. 11, pp. 372-381.

Chan N, Kamberský V, Fraitová D (2000), "Impedance matrix of thin metallic ferromagnetic films and SSWR in parallel configuration," $J$. Magn. Magn. Mater., vol. 214, pp. 93-98.

Chen J, Zhang B, Tang D, Yang Y, Xu W, Lu H (2006), "Possible existence of a new type of left-handed materials in coupled ferromagnetic bilayer films," J. Magn. Magn. Mater., vol. 302, pp. 368-374.

Chang C S, Kostylev M, Ivanov E (2013), " Metallic spintronic thin film as a hydrogen sensor," Appl. Phys. Lett., vol. 102, 142405.

Charilaou M, Lenz K, Kuch W (2010), "Spin-pumping-enhanced magnetic damping in ultrathin $\mathrm{Cu}(001) / \mathrm{Co} / \mathrm{Cu}$ and $\mathrm{Cu}(001) / \mathrm{Ni} / \mathrm{Cu}$ films," J. Magn. Magn. Mater., vol. 322, pp. 2065-2070.

Counil G, Kim J V, Devolder T, Chappert C, Shigeto K, Otani Y (2004), "Spin wave contributions to the high-frequency magnetic response of thin films obtained with inductive methods," J. Appl. Phys., vol. 95, pp. 5646-5652.

Hahn C, de Loubens G, Klein O, Viret M, Naletov V V, Ben Youssef J (2013), "Comparative measurements of inverse spin Hall effects and magnetoresistance in YIG/Pt and YIG/Ta," Phys. Rev. B, vol. 87, 174417.

Jungwirth T, Wunderlich J, Olejník K (2012), "Spin Hall effect devices," Nat. Mater., vol. 11, pp. 382-390.

Kennewell K J, Kostylev M, Ross N, Magaraggia R, Stamps R L, Ali M, Stashkevich A A, Greig D, Hickey B J (2010), "Magnetization pinning at a Py/Co interface measured using broadband inductive magnetometry," J. Appl. Phys., vol. 108, 073917.
Kim D H, Kim H H, Youa C Y (2011), "Suppression of the spin pumping in Pd/Ni81Fe19 bilayers with nano-oxide layer," Appl. Phys. Lett., vol. 99, 072502.

Kostylev M (2009), "Strong asymmetry of microwave absorption by bilayer conducting ferromagnetic films in the microstrip-line based broadband ferromagnetic resonance," J. Appl. Phys., vol. 106, 043903.

Kostylev M, Stashkevich A A, Adeyeye A O, Shakespeare C, Kostylev $\mathrm{N}$, Ross N, Kennewell K, Magaraggia R, Roussigné Y, Stamps R $L$ (2011), "Magnetization pinning in conducting films demonstrated using broadband ferromagnetic resonance," J. Appl. Phys., vol. 108,103914

Kostylev M (2012), "Transmission of microwaves through exchangecoupled bi-layer magnetic films in ferromagnetic and standing spin wave resonances," J. Appl. Phys., vol. 112, 093901.

Kostylev M (2013), "Waveguide-based ferromagnetic resonance measurements of metallic ferromagnetic films in transmission and reflection," J. Appl. Phys., vol. 113, 053908.

Kowalewski M, Butler W H, Moghadam N, Stocks G M, Schulthess T C, Song K J, Thompson J R, Arrott A S, Zhu T, Drewes J, Katti R $R$, McClure M T, Escorcia O (2000), "The effect of Ta on the magnetic thickness of permalloy (Ni81Fe19) films," J. Appl. Phys., vol. 87, 5732 .

Kruglyak V V, Demokritov S O, Grundler D (2010), "Magnonics," J. Phys. D.: Appl. Phys., vol. 43, 264001.

Maksymov I S, Kostylev M (2013), "Impact of conducting nonmagnetic layers on the magnetization dynamics in thin-film magnetic nanostructures," J. Appl. Phys., vol. 113, 043927.

Maksymov I S, Kostylev M (2013a), "Impact of eddy currents on the dispersion relation of surface spin waves in thin conducting magnetic films," J. Phys. D: Appl. Phys., vol. 46, 495001.

Maksymov IS, Kostylev M (2014), "Microwave eddy-current shielding effect in metallic films and periodic nanostructures of sub-skindepth thicknesses and its impact on stripline ferromagnetic resonance spectroscopy," J. Appl. Phys., vol. 116, 173905.

Miroshnichenko A E, Flach S, Kivshar Yu S (2010), "Fano resonances in nanoscale structures," Rev. Mod. Phys., vol. 82, pp. 2257-2298.

Mizukami S, Ando Y, Miyazaki T (2001), "Ferromagnetic resonance linewidth for NM/80NiFe/NM films (NM = Cu, Ta, Pd and Pt)," J. Magn. Magn. Mater., vol. 226-230, pp. 1640-1642.

Sandweg C W, Kajiwara Y, Chumak A V, Serga A A, Vasyuchka V I, Jungfleisch M B, Saitoh E, Hillebrands B (2011), "Spin pumping by parametrically excited exchange magnons," Phys. Rev. Lett., vol. 106, 216601.

Shaw J M, Nembach H T, Silva T J, Boone C T (2013), "Precise determination of the spectroscopic $\mathrm{g}$-factor by use of broadband ferromagnetic resonance spectroscopy," J. Appl. Phys., vol. 114, 243906.

Silva T J, Lee C S, Crawford T M, Rogers C T (1999), "Inductive measurement of ultrafast magnetization dynamics in thin-film Permalloy," J. Appl. Phys., vol. 85, pp. 7849-7862.

Schmiedl E, Wissmann P, Finzel H U (2008), "The electrical resistivity of ultra-thin copper films," Z. Naturforsch., vol. 63a, pp. 739-744

Speriosu V S, Nozieres J P, Gurney B A, Dieny B, Huang T C, Lefakis $H$ (1993), "Role of interfacial mixing in giant magnetoresistance," Phys. Rev. B, vol. 47, pp. 11579-11582.

Stamps R L, Breitkreutz S, Åkerman J, Chumak A V, Otani Y C, Bauer G E W, Thiele J U, Bowen M, Majetich S A, Kläui M, Prejbeanu I L, Dieny B, Dempsey N M, Hillebrands B (2014), "The 2014 magnetism roadmap,” J. Phys. D: Appl. Phys., vol. 47, 333001.

Wu R X (2005), "Effective negative refraction index in periodic metalferrite-metal film composite," J. Appl. Phys., vol. 97, 076105. 